\title{
Safety and Efficiency of Laparoscopic Femoral Hernia Repair with Preserved Uterine Round Ligament
}

\author{
Mingxiao Guo, Dongfeng Chen, Haifeng Zhang and Chunlei Lu \\ Department of General Surgery Center, Linyi People's Hospital, Shandong University, Linyi, China
}

\begin{abstract}
Objective: To evaluate the efficiency and safety of the transabdominal preperitoneal (TAPP) technique with or without preserved uterine round ligament for the repair of femoral hernias (FHs), on the female patients treated at a single centre. Study Design: Comparative-descriptive study.

Place and Duration of Study: Department of General Surgery Center, Linyi People's Hospital, Shandong University, from January 2010 to December 2016.

Methodology: A total of 62 patients were randomly divided into traditional TAPP (T-TAPP) group and modified TAPP (M-TAPP) group, and all the procedures had been successfully completed. The operative time, recurrences and complications were investigated and compared.

Results: Twenty-six patients were randomised into T-TAPP group and 36 patients were randomised into M-TAPP group. There was no obvious difference in terms of age and body mass index between the two groups. The follow-up period was 24 to 90 months. Uterine prolapse occurred in five patients in T-TAPP group, but none in the M-TAPP group. There was significant difference between the two groups $(p<0.05)$. There was also no significant difference in terms of postoperative infection or recurrence of hernia between the two groups nor was there any significant difference between the two groups in development of seroma, post-procedure pain and foreign body sensation during the long-term follow-up.

Conclusion: The modified TAPP is a safe technique with a very low rate of recurrence and a low incidence of chronic pain, which retained the function of the uterine round ligament. It is an ideal method for the treatment of femoral hernia in female.
\end{abstract}

Key Words: Laparoscopic, Femoral hernia, Uterine round ligament, Peritoneum, Recurrences, Complication.

How to cite this article: Guo M, Chen D, Zhang H, Lu C. Safety and efficiency of laparoscopic femoral hernia repair with preserved uterine round ligament in female patients. J Coll Physicians Surg Pak 2019; 29(10):932-6.

\section{INTRODUCTION}

Groin hernias (GHs), including femoral and inguinal hernias, are rare in females compared with the males, with a rough morbidity of $2-5 \%$ in males and $0.3 \%$ in females. The incidence of femoral hernia (FHs) in males is only $2.4 \%$, but which are obligated to $34 \%$ of all groin hernias in females. ${ }^{1-3}$

In recent years, the endoscopic approach via the transabdominal preperitoneal (TAPP) is highly recommended for female patients with primary GHs, if performed by surgeons experienced in laparoscopic technique on the base of the European Hernia Society (EHS) guidelines. ${ }^{4}$ This resulted from the high recurrence rate of femoral after traditional open tension-free herniorrhaphy of groin hernia in woman, which argues for the application of the laparoscopic technique. In addition, TAPP is associated with reduced postoperative pain and early restoration of

Correspondence to: Haifeng Zhang, Department of General Surgery Center, Linyi People's Hospital, 27 East Jiefang Road, Linyi 276000, China.

E-mail:ptwk37@163.com

Received: January 18, 2019; Revised: June 30, 2019;

Accepted: July 31, 2019 normal activities. ${ }^{5,6}$ Therefore, TAPP seems an appealing technique for female with femoral hernia.

All hernias in the groin begin within the myopectineal orifice, which is crossed by femoral vessels as well as the funiculus spermaticus in male or the uterine round ligament in female. In the TAPP approach, the round ligament is transected in approximately half of groin hernia repairs performed, which may be impaired the physiological function of female.7 Nevertheless, personal knowledge and opinions on the function of the ligament and the importance of preserving it varied widely among the experienced surgeons.

Therefore, the rationale of the present study is based upon the physiological function of the uterine round ligament in female. A modified TAPP technique called "uterine round ligament peritoneum", which maintained the integrity of uterine round ligament, has been designed at the study place to provide feedback on the repair of $\mathrm{FHs}$.

The objective of the study was to compare the modified TAPP technique and traditional TAPP techniques of laparoscopic hernia repair in terms of efficiency and safety during follow-up. 


\section{METHODOLOGY}

This study was conducted at the Department of General Surgery Center, Linyi People's Hospital, Shandong University, from January 2010 to December 2016 and approved by the Medical Ethics Committee of Linyi People's Hospital. A total of 62 consecutive patients were enrolled in the study. The clinical diagnosis of a femoral hernia is based on the sac protrudes through the femoral ring into the fossa ovale. ${ }^{8}$ Ultrasound and/or radiological examination was used in case of a dubious swelling and/or obscure pain in the inguinal region, and MRI is preferred when necessary. ${ }^{9}$ The criteria for inclusion in the study were clinical diagnosis of primary unilateral $\mathrm{FHs}$, female patients over 18 years of age, TAPP with mesh fixation, no obvious uterine prolapse, cardiopulmonary, hepatic, or renal impairment, and no operative contraindication. Patients with male gender, acute repairs, open repairs, history of lower abdominal surgery, and bilateral inguinal hernia, recurrent hernia, or incarcerated hernia, and infections of surgical site or bacteremia were excluded from the research.

The cases were recruited by simple random sampling divided into traditional TAPP (T-TAPP) group and modified TAPP (M-TAPP) group, and the distribution ratio is about 1:1.5. Twenty-six patients were randomised into T-TAPP group and 36 patients were randomised into M-TAPP group. Patients in T-TAPP group were treated with conventional TAPP surgery, namely, after intravenous anesthesia induction, endotracheal intubation was performed. Pneumoperitoneum up to $13 \mathrm{mmHg}$ was established with $\mathrm{CO} 2$ at the umbilicus using a Veress needle. A $10 \mathrm{~mm}$ trocar was placed above the umbilicus for insertion of a $30^{\circ}$ laparoscope. After the $\mathrm{FH}$ was identified through the laparoscope, two additional $5 \mathrm{~mm}$ trocars were placed on either side of the umbilicus at the lateral border of the musculus rectus abdominis. The peritoneum was incised $2 \mathrm{~cm}$ above the deep ring from anterosuperior iliac spine to the medial umbilical ligament, to enable the formation of a peritoneal flap. Afterward, the round ligament of uterus was cut off and the Bogros and Retzius spaces were fully exposed and released. After the hernial sac was identified, the traction and contra-traction maneuvers were used for dissection until the sac was reduced. Enough space was subsequently created to accommodate $10 \times 15 \mathrm{~cm}$ polypropylene hernia patch, which was placed to cover the whole myopectineal orifice. Then the mesh was fixed to the pubic tubercle, Cooper ligament, conjoint tendon and posterior sheath of rectus abdominis with biological glue. The peritoneal flap and trocar sites were finally closed with 3-0 absorbable sutures. Same operation was performed, if the $\mathrm{FH}$ was found on the other side.

M-TAPP group patients were treated with the modified TAPP surgery, namely, based on the three-trocar technique as above-mentioned used for the surgical procedure. Through the laparoscope, all anatomical markers were observed and the hernia was confirmed and dissection of a wide peritoneal flap is performed in the usual manner. Afterward, the round ligament of uterus was lifted and dissected widely to create enough space between the round ligament of uterus and the triangle of doom. Once an adequate preperitoneal pocket was made within which to place a $10 \times 15 \mathrm{~cm}$ polypropylene mesh, it was taken to the inguinal region and partial overlapped behind the uterine round ligament, so that the broader part of the hernia patch came to lie medial and the narrower part lies lateral to the uterine round ligament (Figure 1). Then the whole myopectineal orifice on the affected side was covered by the mesh which was fixed in place. Moreover, the part of patch overlapping behind the round ligament of the uterus was fixed to the pelvic wall with biological glue. Afterwards, the peritoneal flap was closed using absorbable sutures.

The demographics, clinical characteristics and operative parameters of the two groups of patients were observed. During the follow-up period, the postoperative complications were assessed by Cleveland-Dindo scales. ${ }^{10}$ A physical check was performed 1-week and 1-month after discharge. The telephone and outpatient follow-up reviews, including recurrence and uterine prolapse, were conducted every 6 months in the first year, and annually thereafter. All data were collected by the computer-based Hospital Information System (HIS) by the third-party professionals. All statistical analyses were performed using the SPSS version 18.0 software (IBM,

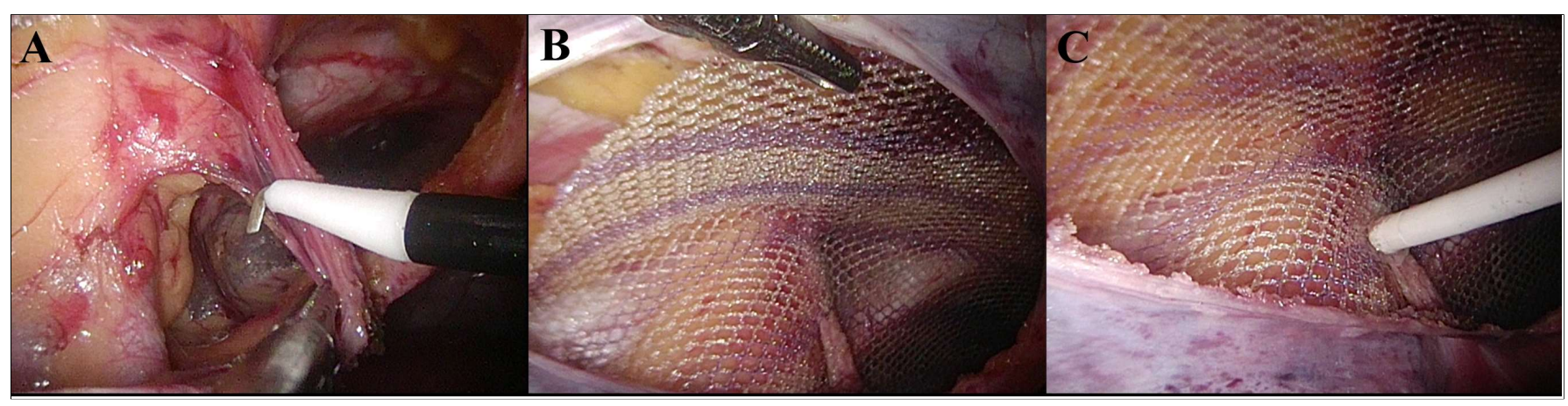

Figure 1: The modified TAPP procedure: (A) Dissected and peritoneal the uterine round ligament free from the pelvic wall; (B) Overlapped the polypropylene mesh behind the uterine round ligament; (C) Fixed the mesh to ligament with fibrin glue. 
Armonk, NY). Shapiro-Wilk test was used for normality of continuous data. Categorical data or dichotomous variables were analysed with the Chi-square test; whereas, student's t-test was used for normal distributed continuous variables. All the factors with $p<0.05$ were considered statistically significant. All data were expressed as mean \pm SD.

\section{RESULTS}

Twenty-six patients were randomised into T-TAPP group and 36 patients were randomised into M-TAPP group. Overall mean age was $42.6 \pm 16.0$ years, ranging from 18-68 years. The average age of patients in T-TAPP group was $43.8 \pm 16.5$ years, ranging from 19-68 years; and that of M-TAPP group, it was $41.8 \pm 15.8$ years; ranging from 18-66 years. The average body mass index (BMI) in T-TAPP group was $24.6 \pm 1.8 \mathrm{Kg} / \mathrm{m}^{2}$ and that of M-TAPP group was $24.2 \pm 2.2 \mathrm{Kg} / \mathrm{m}^{2}$. There was no significant difference between the two groups in terms of age and BMI. The baseline characteristics and demographics of all patients and follow-ups are shown in Table I.

As shown in Table II, there was no injury to the femoral and inferior epigastric vessels, bladder, and bowel

Table I: Demographics, clinical characteristics and follow-ups of all patients.

\begin{tabular}{l|c|c|c}
\hline & T-TAPP Group & M-TAPP Group & p-value \\
\hline Case, $\mathrm{n}(\%)$ & $26(41.9 \%)$ & $36(58.1 \%)$ & 0.253 \\
\hline Age (years) & $43.8 \pm 16.5$ & $41.8 \pm 15.8$ & 0.632 \\
\hline BMI & $24.6 \pm 1.8$ & $24.2 \pm 2.2$ & 0.459 \\
\hline Previous PLAS, $\mathrm{n}(\%)$ & 0 & 0 & \\
\hline Follow-up (months) & $59.2 \pm 21.2$ & $53 \pm 21.9$ & 0.273 \\
\hline
\end{tabular}

Table II: Operative parameters between the two groups.

\begin{tabular}{l|c|c|c}
\hline & $\begin{array}{c}\text { T-TAPP Group } \\
(\mathrm{n}=26)\end{array}$ & $\begin{array}{c}\text { M-TAPP Group } \\
(\mathrm{n}=36)\end{array}$ & -value \\
\hline Operative time (min) & $61.9 \pm 6.5$ & $65.8 \pm 7.3$ & 0.032 \\
\hline $\begin{array}{l}\text { Length of hospital stay } \\
\text { (days) }\end{array}$ & $4.3 \pm 1.1$ & $4.4 \pm 1.1$ & 0.827 \\
\hline Bleeding (mL) & $8.0 \pm 2.3$ & $8.2 \pm 2.6$ & 0.730 \\
\hline concomitant injury & 0 & 0 & \\
\hline Totle Cost $(¥)$ & $12923.1 \pm 452.8$ & $12773.6 \pm 518.0$ & 0.242 \\
\hline
\end{tabular}

during dissection or the insertion of trocar in both groups. Bleeding during operation was graded as minimal in both groups. Although operative time of M-TAPP group is longer than the T-TAPP group, there was no significant difference between the two groups in terms of length of hospital stay, bleeding, and total cost during surgery.

All patients were followed for 24-90 months (average= 61.6 months). Table III shows postoperative complications during the follow-up. Uterine prolapse, seroma formation, infection of mesh or wound, foreign body sensation, recurrence of the hernia, and pain were recorded in follow-up. There were no obvious complications that needed surgical re-intervention. Uterine prolapse occurred in five patients in T-TAPP group, but none in the M-TAPP group. There was significant difference between the two groups $(p<0.05)$. Seroma was the most common problem that was noticed at one week followup and resolved experientially in all patients. There was no significant difference in development of seroma, postprocedure pain, and foreign body sensation between the two groups. There was no recurrence of hernia or wound or mesh infection at 1-month and yearly thereafter.

\section{DISCUSSION}

Femoral herniorrhaphyr is one of the most common elective operations performed by general surgeons, and yet there is no universal consensus on the ideal technique, which could overcome the potential problems of recurrence, infection, and chronic groin pain.10-12 With the development of laparoscopy, TAPP as an alternative to conventional approaches has been embraced wholeheartedly by many general surgeons.

Currently, the advantages of this laparoscopic repair are obvious compared with open techniques.13-16 Firstly, there is minimal trauma to the abdominal wall, resulting in less pain, almost no infection, and a very low rate of hematoma formation. Secondly, no cutting or suturing of fascia structures, complete absence of tension, resulting in less risk of chronic pain. Thirdly, covered the indirect, direct and femoral spaces broadly, reducing the recurrence rate.

Table III: Recurrence and complication parameters during follow-up of the two groups.

\begin{tabular}{l|c|c|c|c}
\hline & Post-operation & $\begin{array}{c}\text { T-TAPP Group } \\
(\mathrm{n}=26) \\
\mathrm{n}(\%)\end{array}$ & $\begin{array}{c}\text { M-TAPP Group } \\
(\mathrm{n}=36) \\
\mathrm{n}(\%)\end{array}$ & $\begin{array}{c}\mathrm{p} \text {-value } \\
\text { Seroma formation (Grade 1)* }\end{array}$ \\
\hline Pain (Grade 2) $^{*}$ & 7-day & $6(23.1 \%)$ & $2(0.5 \%)$ & 0.937 \\
\hline Foreign body sensation & 30-day & $2(0.8 \%)$ & $7(19.4 \%)$ & 0.735 \\
\hline Infection & 7-day & $4(15.4 \%)$ & $6(16.7 \%)$ & 0 \\
\hline Recurrence at 24-90 months & 7-day & $5(19.2 \%)$ & 0 & 0.794 \\
\hline Uterine prolapse & 30-day & 0 & 0 & \\
\hline
\end{tabular}

*Grades were determined following the Clavien-Dindo classification 
The uterine round ligament originates at the uterine horns, enters the pelvis via the deep ring, and passes through the inguinal canal and continues onto the labia majora where its fibers spread and mix with the tissue of the mons pubis. The function of the uterine round ligament is maintenance of the anteversion of the uterus during pregnancy. 17-19 Previously, the uterine round ligament was often transected due to the physiological anatomy in the TAPP approach, which could tilt uterus back, causing uterine prolapse when increased abdominal pressure. ${ }^{7}$ Nevertheless, uterine prolapse not only influences the physiological function, but also produces a series of physiological problems. Therefore, it is essential to maintain the integrity of uterine round ligament. In addition, there are a few scholars relaxed groin hernia and the round ligament of uterus, and these studies are mainly about varicosities and/or cysts on the round ligament mimicking groin hernia during pregnancy.20,21 The personal knowledge and opinions on the function of the ligament and the importance of preserving it varies widely among the experienced surgeons. It appears that the attempt to preserve the ligament depends on individual preferences among surgeons.

In this study, the uterine round ligament was dissected free from the pelvic wall, and preserved covering the abdominal wall broadly by the mesh as flat as possible. The results revealed that uterine prolapse occurred in five patients in T-TAPP group, but none in the M-TAPP group. There was significant difference between the two groups $(p<0.05)$. Of the five patients, 3 were pregnancies and the rest were constipations after herniorrhaphy. Transection of the ligament may increase uterine instability after surgery, and all factors causing high abdominal pressure could lead to uterine prolapse in the long-term follow-up. Although operative time of M-TAPP group is longer than the T-TAPP group, there was no significant difference between the two groups in terms of pain, seroma formation, infection of mesh or wound, foreign body sensation and the hernia recurrence during the long-term follow-up. This showed that, although preserving the ligament slightly prolonged the operation time, it did not affect the recovery of patients after operation. At the same time, on the basis of retaining the function of uterine round ligament, the expected effect of hernia repair was achieved.

In our paper, we achieved satisfactory results through modified method. Moreover, combined with our experience and literature summary, the operation skills are summarised as follows: Firstly, dissected and peritoneal the uterine round ligament full free from the pelvic wall. Secondly, cut a small hole at the lowest point of the mesh that forms the new profound inguinal ring, and overlapped and fixed the mesh behind the uterine round ligament. Lastly, the key for success of this technique was satisfactory overlaps of the mesh part behind the round ligament of the uterus and proper fixation besides the implantation of patch.

\section{CONCLUSION}

There was no significant difference in intraoperative parameters between T-TAPP and M-TAPP group, nor was there any obvious difference between the two groups in terms of postoperative complication during the long-term follow-up. But, the modified transabdominal preperitoneal inguinal hernia repair is a safe technique with a very low rate of chronic pain and recurrence, which retained the function of the uterine round ligament. It is an ideal method for the treatment of femoral hernia in female.

\section{ACKNOWLEDGEMENTS:}

This study was supported by National Natural Science Foundation of China (Grant No. 81500688), Shandong Provincial Natural Science Foundation of China (Grant No. ZR2015HL033) and Shandong Provincial Medical and Health Science and Technology Development program (Grant No. 2016WS0237).

\section{ETHICAL APPROVAL:}

This work was approved by the Medical Ethics Committee of Linyi People's Hospital and the ethical approvals were obtained prior to initiation of the research work.

\section{PATIENTS' CONSENT:}

Written informed consents on the protocol were obtained from patients to publish the data concerning this case.

\section{CONFLICT OF INTEREST:}

The authors have no conflict of interest regarding the content of this article.

\section{AUTHORS' CONTRIBUTION:}

DC, MG: Data curation and analysis.

MG: Written original draft.

$\mathrm{CL}, \mathrm{HZ}$ : Reviewed and edited.

\section{REFERENCES}

1. Köckerling F, Simons MP. Current concepts of inguinal hernia repair. Visc Med 2018; 34:145-50.

2. He Z, Hao X, Feng B, Li J, Sun J, Xue P, et al. Laparoscopic repair for groin hernias in female patients: A single-center experience in 15 years. J Laparoendosc Adv Surg Tech A 2019; 29:55-9.

3. Köckerling F, Lorenz R, Hukauf M, Grau H, Jacob D, Fortelny R, et al. Influencing factors on the outcome in female groin hernia repair: A registry-based multivariable analysis of 15,601 patients. Ann Surg 2019; 270:1-9.

4. Hernia Surge Group. International guidelines for groin hernia management. Hernia 2018; 22:1-165.

5. Chen D, Su N, Wang W, Zhang Z, Guo M, Lu C, et al. Laparoscopic transabdominal preperitoneal technique versus open 
surgery with the ultrasound hernia system for the repair of female primary femoral hernias - An observational retrospective study. Medicine (Baltimore) 2018; 97:e13575.

6. Langeveld HR, van't Riet M, Weidema WF, Stassen LPS, Steyer-berg EW, Lange J, et al. Total extraperitoneal inguinal hernia repair compared with Lichtenstein (the level-trial): A randomized controlled trial. Ann Surgery 2010; 251:819-24.

7. Schmidt L, Andresen K, Öberg S, Rosenberg J. Dealing with the round ligament of uterus in laparoscopic groin hernia repair: A nationwide survey among experienced surgeons. Hernia 2018; 22:849-55.

8. Fränneby U, Gunnarsson U, Andersson M, Heuman R, Nordin P, Nyrén $\mathrm{O}$, et al. Validation of an inguinal pain questionnaire for assessment of chronic pain after groin hernia repair. $\mathrm{Br} J$ Surg 2008; 95:488-93.

9. Simons MP, Aufenacker T, Bay-Nielsen M, Bouillot JL, Campan-elli G, Conze J, et al. European hernia society guidelines on the treatment of inguinal hernia in adult patients. Hernia 2009; 13:343-403.

10. Dindo D, Demartines N, Clavien PA. Classification of surgical complications: A new proposal with evaluation in a cohort of 6336 patients and results of a survey. Ann Surg 2004; 240: 205-13.

11. Goethals A, Adams CT. Femoral hernia. Stat pearls [Internet]. Treasure Island:FL; 2018.

12. Al-Kandari A. Is laparoscopic inguinal hernia repair more effective than open repair? J Coll Physicians Surg Pak 2011; 21:291-6.

13. Salma U, Ahmed I, Ishtiaq S. A comparison of postoperative pain and hospital stay between Lichtenstein's repair and laparoscopic transabdominal preperitoneal (TAPP) repair of inguinal hernia: A randomized controlled trial. Pak J Med Sci 2015; 31:1062-6.

14. Klobusicky P, Hoskovec D. Reduction of chronic postherniotomy pain and recurrence rate. Use of the anatomical self-gripping ProGrip laparoscopic mesh in TAPP hernia repair. Preliminary results of a prospective study. Wideochir Inne Tech Maloinwazyjne 2015; 10:373-81.

15. Bernhardt GA, Gruber G, Molderings BS, Cerwenka H, Glehr M, Giessauf $\mathrm{C}$, et al. Health-related quality of life after TAPP repair for the sportsmen's groin. Surg Endosc 2014; 28:439-46.

16. Scheuermann U, Niebisch S, Lyros O, Jansen-Winkeln B, Gockel I. Transabdominal preperitoneal (TAPP) versus Lichtenstein operation for primary inguinal hernia repair A systematic review and meta-analysis of randomized controlled trials. BMC Surg 2017; 17:55.

17. Gui B, Valentini AL, Ninivaggi V, Marino $M$, lacobucci $M$, Bonomo L. Deep pelvic endometriosis: Don't forget round ligaments. Review of anatomy, clinical characteristics, and MR imaging features. Abdom Imaging 2014; 39:622-32.

18. Acién P, Sánchez del Campo F, Mayol MJ, Acién M. The female gubernaculum: Role in the embryology and development of the genital tract and in the possible genesis of malformations. Eur J Obstet Gynecol Reprod Biol 2011; 159:426-32.

19. Tolver MA, Strandfelt P, Rosenberg J, Bisgaard T. Female gender is a risk factor for pain, discomfort, and fatigue after laparoscopic groin hernia repair. Hernia 2013; 17:321-7.

20. Lechner M, Fortelny R, Ofner D, Mayer F. Suspected inguinal hernias in pregnancy - handle with care! Hernia 2014; 18:375-9.

21. Polat AV, Aydin R, Polat AK, Kececi IS, Karahan G, Taskin GO. Round ligament varicosities: A rare cause of groin swelling in pregnancy. Abdom Imaging 2013; 38:1178-81. 